\title{
Compartilhar para Alcançar: Desenvolvimento Web Acessível na Prática, no Dia a Dia
}

\author{
Vagner Figueredo \\ de Santana \\ IBM Research \\ IBM \\ São Paulo, Brasil \\ vagsant@br.ibm.com
}

\author{
Leonelo Dell \\ Anhol Almeida \\ Universidade Tecnológica \\ Federal do Paraná (UTFPR), \\ Curitiba, Brasil \\ leoneloalmeida@utfpr.edu.br
}

\author{
Maria Cecília Calani \\ Baranauskas \\ Universidade Estadual de \\ Campinas (UNICAMP) \\ Campinas, Brasil \\ cecilia@ic.unicamp.br
}

\begin{abstract}
APRESENTAÇÃO
As diretrizes de acessibilidade de conteúdo (WCAG 2.0) do World Wide Web Consortium (W3C) completam neste ano 10 anos com o status de recomendação. Entretanto, a Web ainda conta com muitas barreiras relacionadas a problemas básicos de codificação. Estudo aponta que $85 \%$ dos websites apresentam problemas como falta de rótulos em formulários e de descrição textual para imagens [1]. Outro ponto crítico é que nem sempre profissionais sabem como converter diretrizes em ações. Neste minicurso apresentaremos resultados relacionados ao projeto WARAU (Websites Atendendo a Requisitos de Acessibilidade e Usabilidade). $\mathrm{O}$ projeto teve início em 2008 e envolveu o estudo sobre os desafios que pequenas equipes de desenvolvimento enfrentam ao envolver aspectos relacionados à acessibilidade e usabilidade no dia a dia de suas atividades. O projeto resultou em um livro publicado recentemente [2] que poderá ser obtido gratuitamente pelos participantes do minicurso. $\mathrm{O}$ minicurso envolverá dinâmicas e exposição de cenários de criação e avaliação de conteúdo web acessível.
\end{abstract}

\section{SUMÁRIO ESTENDIDO}

$\mathrm{O}$ projeto Websites Atendendo a Requisitos de Acessibilidade e Usabilidade, ou WARAU, nasceu como um website que tinha o objetivo de ser um espaço de discussão sobre normas, diretrizes, técnicas e boas práticas para a criação de código Web acessível e usável. O projeto também envolveu um estudo realizado com uma equipe de desenvolvimento de sistemas Web da UNICAMP em que foram identificadas dificuldades e desafios relacionados ao desenvolvimento Web acessível combinando HTML, CSS, JavaScript, Acessibilidade e Usabilidade. Hoje, o conteúdo do WARAU está disponibilizado no formato de livro [2]. $\mathrm{O}$ projeto considera diferentes perfis de autores na Web:

Permission to make digital or hard copies of all or part of this work for personal or classroom use is granted without fee provided that copies are not made or distributed for profit or commercial advantage and that copies bear this notice and the full citation on the first page. Copyrights for components of this work owned by others than the author(s) must be honored. Abstracting with credit is permitted. To copy otherwise, or republish, to post on servers or to redistribute to lists, requires prior specific permission and/or a fee. Copyright 2018 SBC.

IHC 2018, Anais Estendidos do XVII Simpósio Brasileiro sobre Fatores Humanos em Sistemas Computacionais

Outubro 22-26, 2018, Belém, Brasil

Minicursos. desenvolvedor, designer e redator. Ele é especialmente relevante para mantenedores de websites que queiram aprender como construir websites válidos, acessíveis e usáveis, a partir de exemplos. A seguir apresentamos os princípios resultantes do projeto e que serão abordados durante o minicurso.

Padrões de codificação: Este princípio envolve tarefas cotidianas da equipe e como especificar/seguir convenções simples de nomenclatura e escrita de código melhoram a legibilidade de código como um todo e facilitam correção e inclusão de novos recursos.

Reaproveitamento de código: Este princípio considera que todo o código Web deve ser escrito de tal forma que possa ser reaproveitado, seja HTML, CSS ou JavaScript, aumentando consistência e reduzindo de consumo de recursos.

Semântica no código: Este princípio realça que linguagens de marcação contam com semântica específica e considerála muitas vezes reduz barreiras de acessibilidade mais comuns.

Aplicar padrões e diretrizes: Este princípio busca relembrar que existem padrões/diretrizes que solucionam grande parte dos problemas comumente encontrados em websites.

Não se restringir a padrões e diretrizes: Este princípio complementa o anterior ao pontuar que, apesar de trazerem melhorias, padrões e diretrizes não são soluções suficientes para a garantia de qualidade de um website. Para tanto é necessário considerar as condições e restrições de uso específicas de cada website e seu público-alvo.

Considerar a diversidade de usuários: Ao contrário do que geralmente é adotado por mantenedores de websites, desenvolver websites para o "usuário médio" não é garantia de ampla aceitação de websites. Portanto, o conhecimento da diversidade de usuários pode ser fator determinante para o sucesso de um website. Tal conhecimento complementa e, por vezes, redireciona diretrizes e padrões.

Considerar diferentes formas de apresentação de páginas Web (dispositivos e configurações): Websites não são documentos estáticos e, portanto, seriam mais bem construídos se fossem considerados como construções 
flexíveis e adequados a diferentes dispositivos, tamanho de display e preferências de visualização de usuários.

Avaliação e validação: Devido à característica dinâmica de websites, mesmo quando mantenedores conhecem e empregam os padrões e recomendações, a tarefa de manter um website atendendo completamente a essas recomendações exige um monitoramento constante. Esse monitoramento pode ser obtido por meio de ferramentas automatizadas de validação de código como parte do processo de desenvolvimento/homologação ou por meio de avaliação manual.

Integrar tecnologias e conceitos durante todo o desenvolvimento: $\mathrm{Um}$ dos grandes problemas no desenvolvimento de websites é a lacuna entre as recomendações técnicas e os conceitos relacionados. Um exemplo disso é a recomendação de acessibilidade sobre o fornecimento de texto alternativo a imagens. Apesar de proverem texto alternativo e, portanto, seguirem a diretriz em questão, mantenedores falham na escolha de qual informação deveria estar presente nesse texto e comumente não sabem quais são os usuários que se beneficiam desse recurso. Para tanto é necessária uma abordagem integrada que permita a compreensão não somente das regras de desenvolvimento, mas também das necessidades e dos benefícios gerados por sua aplicação.

\section{PÚBLICO-ALVO}

Alunos de graduação e pós-graduação que atuem como desenvolvedor, designer e/ou redator com conhecimentos básicos em tecnologias Web (e.g., HTML, CSS, JavaScript).

\section{BIOGRAFIA DOS AUTORES}

\section{Vagner Figueredo de Santana}

Possui graduação em Ciência da Computação - Bacharelado pela Universidade Presbiteriana Mackenzie (2006), mestrado em Ciência da Computação pela UNICAMP (2009) e doutorado em Ciência da Computação pela Universidade Estadual de Campinas (UNICAMP) (2012). Durante o doutorado visitou o laboratório Human Interface in Information Systems, do Consiglio Nazionale delle Ricerche, em Pisa, Itália. Lecionou disciplinas relacionadas à Engenharia de Software como professor visitante na pósgraduação da Universidade Presbiteriana Mackenzie de 2009 a 2015. Foi webmaster da Folha de S.Paulo (www.folha.com.br) de 2002 a 2007. É pesquisador e Master Inventor na IBM Research desde 2012. Também atua como docente colaborador na Pós-Graduação em Ciência da Computação da Universidade Federal do ABC (UFABC), onde orienta alunos de mestrado. Tem experiência na área de Ciência da Computação, com ênfase em tecnologias Web e Interação Humano-Computador, com ênfase em avaliação de Interfaces de Usuário e análise de $\log s$ de interação com foco em acessibilidade e usabilidade.

\section{Leonelo Dell Anhol Almeida}

Possui graduação em Bacharelado em Informática pela Universidade Estadual de Ponta Grossa (2003), mestrado em
Informática pela Universidade Federal do Paraná (2006) e doutorado em Ciência da Computação pela Universidade Estadual de Campinas (2011). Atualmente é professor adjunto do magistério superior da Universidade Tecnológica Federal do Paraná. Atua no Programa de Pós-Graduação em Computação Aplicada (PPGCA, UTFPR), no nível de mestrado, e no Programa de Pós-Graduação em Tecnologia (PPGTE, UTFPR), nos níveis de mestrado e de doutorado. Tem experiência na área de Ciência da Computação, com ênfase em Interação Humano-Computador, atuando principalmente nos seguintes temas: acessibilidade, Design de Interação, estudos em Ciência, Tecnologia e Sociedade e sistemas colaborativos.

\section{Maria Cecília Calani Baranauskas}

Possui graduação em Ciência da Computação - Bacharelado pela Universidade Estadual de Campinas (1976) e em Matemática - Licenciatura pela Universidade Estadual de Campinas (1976), mestrado em Ciência da Computação pela Universidade Estadual de Campinas (1981) e doutorado em Engenharia Elétrica pela Universidade Estadual de Campinas (1993). Realizou pós-doutoramento no Semiotics Special Interest Group (SSIG) na Staffordshire University (School of Computing) em 2001 e no Applied Informatics with Semiotics (AIS) lab da University of Reading (Dept. of Computer Science) em 2002, UK. Recebeu a Cátedra IberoAmericana UNICAMP-Santander Banespa para estudar problemas de acessibilidade em engenharia de software na Universidad Politécnica de Madrid, Espanha (2006-2007). Foi agraciada com o Diploma do Mérito Educacional "Prof. Darcy Ribeiro" em 2006 e com o ACM SIGDOC Rigo Award em 2010. É Professora Titular da Universidade Estadual de Campinas. Tem experiência na área de Ciência da Computação, com ênfase em Metodologia e Técnicas da Computação, atuando principalmente nos seguintes temas: interação humano-computador, semiótica organizacional, interface de usuário e design de sistemas computacionais em diversos domínios (social, educacional, de trabalho).

\section{DURAÇÃO DO MINICURSO}

Três horas.

\section{IDIOMA}

Português.

\section{INFRAESTRUTURA}

Projetor, caixas de som e flipchart com marcador.

\section{REFERÊNCIAS}

1. Vagner Figueredo de Santana e Rogério Abreu de Paula. 2013. Web accessibility snapshot: an effort to reveal coding guidelines conformance. In Proceedings of the 10th International Cross-Disciplinary Conference on Web Accessibility (W4A '13). ACM, New York, NY, USA, Article 2, 4 pages.

2. Vagner Figueredo de Santana, Leonelo Dell Anhol Almeida e Maria Cecilia Calani Baranauskas. 2018. Websites Atendendo a Requisitos de Acessibilidade e Usabilidade. Leanpub. https://leanpub.com/warau/ 